\title{
Cost-effectiveness of KRAS, EGFR and $A L K$ testing for decision making in advanced nonsmall cell lung carcinoma: the French IFCT-PREDICT.amm study
}

\author{
Sandrine Loubière ${ }^{1,2}$, Alexandre Drezet ${ }^{1}$, Michèle Beau-Faller ${ }^{3}$, \\ Denis Moro-Sibilot ${ }^{4}$, Sylvie Friard ${ }^{5}$, Marie Wislez ${ }^{6}$, Hélène Blons ${ }^{7}$, \\ Catherine Daniel $^{8}$, Virginie Westeel ${ }^{9}$, Anne Madroszyk ${ }^{10}$, Hervé Léna ${ }^{11}$ \\ Patrick Merle $^{12}$, Julien Mazières ${ }^{13}$, Gérard Zalcman ${ }^{14,15}$, Roger Lacave ${ }^{16}$, \\ Martine Antoine ${ }^{17}$, Franck Morin ${ }^{18}$, Pascale Missy ${ }^{18}$, Fabrice Barlesi ${ }^{19}$, \\ Pascal Auquier ${ }^{1,2}$ and Jacques Cadranel ${ }^{6}$ on behalf of the French Cooperative \\ Thoracic Intergroup (IFCT)
}

@ERSpublications

KRAS, EGFR and ALK molecular testing before treatment initiation proves to be cost-effective in advanced NSCLC http://ow.ly/Ld1Z30ia9qv

Cite this article as: Loubière $\mathrm{S}$, Drezet $\mathrm{A}$, Beau-Faller $\mathrm{M}$, et al. Cost-effectiveness of KRAS, EGFR and ALK testing for decision making in advanced nonsmall cell lung carcinoma: the French IFCT-PREDICT.amm study. Eur Respir J 2018; 51: 1701467 [https://doi.org/10.1183/13993003.01467-2017].

ABSTRACT ALK rearrangement and EGFR/KRAS mutations constitute the primary biomarkers tested to provide targeted or nontargeted therapies in advanced nonsmall cell lung cancer (NSCLC) patients. Our objective was to assess the cost-effectiveness of biomarker testing for NSCLC.

Between 2013 and 2014, 843 treatment-naive patients were prospectively recruited at 19 French hospitals into a longitudinal observational cohort study. Two testing strategies were compared, i.e. with "at least one biomarker status known" and "at least KRAS status known", in addition to "no biomarker testing" as the reference strategy. The Kaplan-Meier approach was employed to assess restricted mean survival time. Direct medical costs incurred by hospitals were estimated with regard to treatment, inpatient care and biomarker testing.

Compared with "no biomarker testing", the "at least one biomarker status known" strategy yielded an incremental cost-effectiveness ratio of EUR13230 per life-year saved, which decreased to EUR7444 per life-year saved with the "at least KRAS status known" testing strategy. In sensitivity analyses, biomarker testing strategies were less costly and more effective in $41 \%$ of iterations.

In summary, molecular testing prior to treatment initiation proves to be cost-effective in advanced NSCLC management and may assist decision makers in defining conditions for further implementation of these innovations in general practice. 
Affiliations: 'Service Epidémiologie et Economie de la Santé, Assistance Publique Hôpitaux de Marseille, Marseille, France. ${ }^{2}$ Faculté de Médecine - Secteur Timone, Aix-Marseille Université, EA 3279 CEReSS, Marseille, France. ${ }^{3}$ Laboratoire de Biochimie et de Biologie Moléculaire et Plateforme de Génomique des Cancers, Centre Hospitalier Universitaire de Hautepierre, EA3430, Strasbourg. France. ${ }^{4}$ Unité d'Oncologie Thoracique, Clinique de Pneumologie, Centre Hospitalier Universitaire Grenoble-Alpes, Grenoble, France. ${ }^{5}$ Service de Pneumologie, Hôpital Foch, Suresnes, France. ${ }^{6}$ Service de Pneumologie, Assistance Publique Hôpitaux de Paris, Hôpital Tenon, Sorbonne Universités, UPMC Université Paris 06, GRC 04, Theranoscan, Paris, France. ${ }^{7}$ Service de Biochimie, Pharmacogénétique, Oncologie Moléculaire, INSERM UMR-S1147, Université Sorbonne Paris Cité, Assistance Publique Hôpitaux de Paris, Hôpital Européen Georges Pompidou (HEGP), Paris, France. ${ }^{8}$ Oncologie Médicale, Institut Curie, Paris, France. ${ }^{9}$ Service de Pneumologie, CHU Besançon, Hôpital Jean Minjoz, Besançon, France. ${ }^{10}$ Service d'Oncologie Médicale, Institut Paoli Calmettes, Marseille, France. ${ }^{11}$ Centre Hospitalier Universitaire Rennes, Hôpital Pontchaillou, Rennes, France. ${ }^{12}$ Service de Pneumologie, Centre Hospitalier Universitaire de Clermont-Ferrand, Clermont-Ferrand, France. ${ }^{13}$ Service de Pneumologie, Pôle Voies Respiratoires, Centre Hospitalier Universitaire de Toulouse, Hôpital Larrey, Toulouse, France. ${ }^{14}$ Service de Pneumologie et d'Oncologie Thoracique, CHU Caen, Côte de Nacre, Caen, France. ${ }^{15}$ Service d'Oncologie Thoracique, Hôpital Bichat, Paris, France. ${ }^{16}$ Service Histologie - Embryologie Cytogénétique Biologie Tumorale et Génétique Moléculaire, Assistance Publique Hôpitaux de Paris, Hôpital Tenon, Paris, France. ${ }^{17}$ Service d'Anatomie et Cytopathologie Paris, Assistance Publique Hôpitaux de Paris, Hôpital Tenon, Paris, France. ${ }^{18}$ Unité de Recherche Clinique, Intergroupe Francophone de Cancérologie Thoracique (IFCT), Paris, France. ${ }^{19}$ Dépt Oncologie Multidisciplinaire et Innovations Thérapeutiques, AixMarseille Université, Assistance Publique Hôpitaux de Marseille, Hôpital Nord, Centre d'Investigation Clinique, Marseille, France.

Correspondence: Sandrine Loubière, Faculté de Médecine - Secteur Timone, Aix-Marseille Université, EA 3279 CEReSS, 27 Boulevard Jean Moulin, 13005 Marseille Cedex 5, France. E-mail: sandrine.loubiereduniv-amu.fr

\section{Introduction}

Recent advances in identifying oncogenic drivers in nonsmall cell lung cancer (NSCLC) have resulted in strategies designed to further personalise therapy for individual patients based on specific biomarker testing with appropriate targeted or nontargeted therapies [1-3]. These therapeutic approaches referred to anticancer therapies based on tyrosine kinase inhibitors (TKIs). Epidermal growth factor receptor (EGFR) gene mutations were the first targets for TKI treatment in NSCLC. Erlotinib (Tarceva) or gefitinib (Iressa), specific EGFR TKIs, and more recently afatinib (Giotrif) have been shown to improve progression-free and overall survival compared with standard treatment with platinum-based chemotherapy for all NSCLC histologies [4-6]. However, the vast majority of patients acquire resistance and show relapses to EGFR TKIs. On the contrary, the presence of KRAS (KRAS proto-oncogene) mutation or EGFR exon 20 mutation seems to be associated with primary resistance to EGFR-TKI targeted therapy, although the extent to which these might influence treatment selection remains somewhat unclear [7-9]. More recently, the description of EML4 (echinoderm microtubule-associated protein like 4)-ALK (anaplastic lymphoma kinase) fusion as a signalling pathway driver has endorsed the development of the specific TKIs crizotinib (Xalkori) and ceritinib (Zykadia) [10]. Crizotinib showed a $61 \%$ overall response rate in pre-treated patients with this rearrangement [11] and its superiority compared with standard first-line pemetrexed platinum doublet chemotherapy in patients with $A L K$ rearrangement has been recently demonstrated [12].

Recent studies comparing exposed and nonexposed patients with oncogenic drivers to the appropriate therapy confirm the effectiveness of such "biomarker testing and treatment" strategies in improving overall survival [13]. That said, few economic studies have been published to date assessing the cost-effectiveness of such strategies compared with the "no testing and treatment strategy" [14-18].

The presence of EGFR/KRAS mutations and $A L K$ rearrangements is mutually exclusive in patients, and awareness of at least one of these molecular alterations prior to therapy initiation may help optimise the algorithm of NSCLC management care for selecting the most effective therapeutic agents. The question of the interest of a step-by-step approach remains relevant, in France but also in all countries that have a testing policy in the general population. Next-generation sequencing (NGS) strategies, making a step-by-step approach useless, are being deployed throughout the French territory and European countries, but not everywhere at the same pace. Furthermore, this approach does not address the issue of screening for $A L K$ rearrangements. Finally, the diagnostic accuracy of immunohistochemistry (IHC) for $A L K$ is not perfect and some centres perform a validated fluorescence in situ hybridisation (FISH) assay to confirm negative results with $A L K$ IHC, after getting negative results of NGS testing. By comparing routine biomarker testing strategies with the no testing strategy in terms of their relative benefits and costs, cost-effectiveness analyses can serve as a key element to inform decision makers and better define public health policy [19].

To the best of our knowledge, only one single economic analysis has previously evaluated predictive screening of biomarkers in NSCLC, using a micro-simulation model with clinical outcomes, probabilities of disease progression and cost parameters assessed based on various sources and hypotheses [18]. The 
objective of our study was to investigate the cost-effectiveness of routine biomarkers testing prior to initiating NSCLC treatment in common practice in a prospective patient cohort. The PREDICT.amm study enrolled all consecutive, previously untreated, advanced-stage NSCLC patients eligible for first-line therapy from 19 French regional thoracic oncology centres. We performed a cost-effectiveness analysis in terms of costs borne by the healthcare system by evaluating and comparing: 1) the potential gain in survival, and 2) the costs of testing, treatment and management care using biomarker testing strategies versus standard of care therapy without biomarker testing.

\section{Material and methods}

Ethics statement

This study was approved by a National Ethics Committee, the French Advisory Committee on Information Processing in Research in the Field of Health, and the National Commission of Informatics and Freedoms, in compliance with French legislation. All subjects provided informed consent to join the study.

\section{Study subjects}

Consecutive eligible subjects aged $\geqslant 18$ years, treatment naive and affected by advanced-stage NSCLC were recruited between January 2013 and February 2014. All patients underwent molecular profiling performed by one of the 28 regional French National Cancer Institute-certified molecular genetics centres. The routine molecular screening adhered to the national recommendations for advanced nonsquamous NSCLC, including KRAS and EGFR mutations, ALK rearrangements, and two emerging biomarkers, i.e. $B R A F$ (B-Raf proto-oncogene) and HER2 (human EGFR2) mutations [3]. Patients presenting with other tumour types (e.g. squamous cell and mixed histology) could be screened upon approval by their local multidisciplinary tumour board.

\section{Molecular analysis}

First, the pathologist of each participating centre completed the IHC analysis to specify the histological subtypes (adenocarcinoma, squamous cell carcinoma, large cell carcinoma, not otherwise specified) and provided a haematoxylin/eosin stain for molecular analysis. The strategies to identify the ALK rearrangement in the PREDICT.amm cohort were: 1) ALK IHC first, then a validation by FISH assay for IHC-positive cases [20], and 2) simultaneous ALK IHC and FISH testing. The molecular analysis strategies performed in the cohort were: 1) simultaneous testing of all EGFR (exons 18-21), KRAS (exons 2 and 13), BRAF (V600) and HER2 (exon 20) exons, and 2) targeted analysis for KRAS first, then EGFR, followed by the confirmation of mutations using either targeted or nontargeted techniques according to the laboratory's experience. In the latter strategy, each of the other molecular alterations was assessed until a mutation was found, starting with the most common (EGFR mutations) and ending with the least frequent (HER2 mutations).

Each molecular genetics centre employed either the Sanger sequencing method or a more sensitive, validated, allele-specific technique to assess EGFR (exons 18-21) and KRAS (exon 2) mutations [21-23].

The molecular genetics centres sent their results to each medical investigator by means of a specific data sheet. The data were recorded and monitored by the French Cooperative Thoracic Intergroup (IFCT).

\section{Strategies compared}

Three strategies were compared, i.e. a "no biomarker testing" approach with empirical, nonguided treatment based on clinical and pathological parameters, and two different testing strategies in which an appropriate treatment was initiated following concurrent biomarker tests. In one such strategy, i.e. that with "at least one biomarker testing", the result was known at the time of first- or second-line treatment initiation, thus leading to initiation of the most appropriate treatment, whether platinum-based chemotherapy (cisplatin or carboplatin and docetaxel, gemcitabine, paclitaxel, pemetrexed or vinorelbine), platinum doublet chemotherapy plus bevacizumab, nonplatinum doublet chemotherapy or TKI. Concerning this testing strategy, patients received molecular-guided treatment based on either EGFR and KRAS mutations or ALK rearrangement status. First- and second-line therapies were considered together to ensure the inclusion of all patients receiving a targeted therapy, such as crizotinib based on ALK translocation testing, in the molecular-guided treatment group. In the other strategy, i.e. that with "at least KRAS test result known", at the time of first-line treatment, patients received molecular-guided treatment based on KRAS mutation testing, with either EGFR or ALK testing results for characterising patients with tumours harbouring wild-type KRAS (on the basis of KRAS exon 2, EGFR exons 18-21 and ALK FISH analyses). We focused on this specific testing strategy for the following reasons: KRAS mutation is the most frequent [24] and mutations are exclusive; therefore, performing KRAS testing would potentially save EGFR and $A L K$ testing, and would also allow for economy of targeted therapies. The "no biomarker testing" strategy included all patients for whom testing results were not available at the time of first- or 
second-line treatment initiation for the following reasons: tissue not available, technical failure, result not recovered and result not available on time for the therapeutic decision, although some were found positive later for $A L K, K R A S$ or EGFR mutation. The "no biomarker testing" group received all conventional treatments defined above except TKIs.

\section{Cost and effectiveness assessment}

We performed cost-effectiveness analyses based on the healthcare payer's perspective. Incremental cost-effectiveness ratios (ICERs) were calculated and compared between the two groups, corresponding to the difference in mean costs (expressed in EUR) divided by the difference in mean effectiveness (i.e. the between-group difference in restricted mean survival time). ICERs were expressed in terms of costs per life-year saved, in accordance with the Consolidated Health Economic Evaluation Reporting Standards guidelines for economic evaluation [25]. Survival was calculated as the time (in months) from the date of first-line treatment initiation to patient death due to any given cause. Patients still alive were censored at the date of the last follow-up visit. The cut-off date was July 31, 2015, precisely 17 months after enrolling the last patient.

The economic analysis considered all direct healthcare costs in relation to testing, treatment and management care. All input data were collected individually and prospectively for each patient enrolled into the study. The data included costs relating to molecular assays, TKIs, pemetrexed and bevacizumab, acquisition and administration of chemotherapies (including inpatient stay and chemotherapy sessions), as well as to inpatient care for managing adverse events, disease progression and NSCLC disease surveillance (table 1).

Unit costs were obtained from the French national health insurance system and inpatient costs derived for valuation of diagnosis-related groups (DRGs), based on 2015 hospital activity and associated expenditures (Agence Technique de l'Information sur l'Hospitalisation: www.atih.sante.fr). As the follow-up time horizon was short, discounting was not applied.

\section{Statistical and sensitivity analyses}

One-to-one propensity-score-matching approaches were performed in order to minimise confounding factors in terms of survival and cost analyses owing to the study design [26]. Clinical characteristics upon enrolment were analysed using either Kruskal-Wallis, Chi-squared, Fisher's exact or t-tests, as appropriate, in order to identify those characteristics that may influence cost and effectiveness outcomes. Propensity scores were computed for each patient by means of age, sex, body mass index (BMI), tumour/node/ metastasis (TNM) stage, performance status, metastasis localisation, adenocarcinoma histology and smoking history as covariates for sample matching in order to balance between molecular-guided treatment groups and the "no biomarker testing" group. Mean restrictive survival time was determined via the Kaplan-Meier method and compared using the log-rank test. Statistical significance was defined as $\mathrm{p}<0.05$. Immortal time bias was not observed in our observational cohort design as cohort entry was defined at time of first-line treatment initiation, and no statistically significant difference was observed in length of time to diagnosis and first-line treatment steps between the two groups [27].

One-way deterministic sensitivity analyses were carried out in order to identify the main driver parameters of the ICER. The estimate for a given parameter was altered, keeping the other parameters constant, within a range of likely values derived from $95 \%$ confidence intervals. Tornado diagrams were drawn to represent the maximum variation of ICER for sampling variables. A Monte Carlo micro-simulation with 10000 replications was provided in a radar screen format, where the $x$-axis shows the difference in effectiveness and the $y$-axis shows the difference in costs between strategies. In addition, cost-effectiveness acceptability curves were constructed to represent decision uncertainty surrounding cost-effectiveness thresholds [28, 29]. R (www.r-project.org) and TreeAge Pro 2011 (www.treeage.com) were utilised for statistical and sensitivity analyses, respectively.

\section{Results}

Baseline characteristics of all study patients and in matched populations

843 treatment-naive advanced NSCLC patients were enrolled into the study. Overall, 41 patients were excluded from the analysis (protocol deviations, $\mathrm{n}=38$; death, $\mathrm{n}=1$; patient's report not assessable, $\mathrm{n}=2$ ) (figure 1). Finally, a total of 647 patients were included in the "at least one biomarker testing" group and compared with 155 patients in the "no biomarker testing" group. In a second analysis, all patients from the "at least KRAS test result known" group for whom KRAS mutational status was not explored at the time of first-line therapy $(\mathrm{n}=177)$ were excluded. Then, 470 patients were included in the "at least KRAS test result known" intervention and compared with the same 155 control patients. The two groups did not differ significantly in terms of age, sex ratio and TNM stage (supplementary table E1). The intervention 
TABLE 1 Unit costs (base case value and low/high value for the sensitivity analysis)

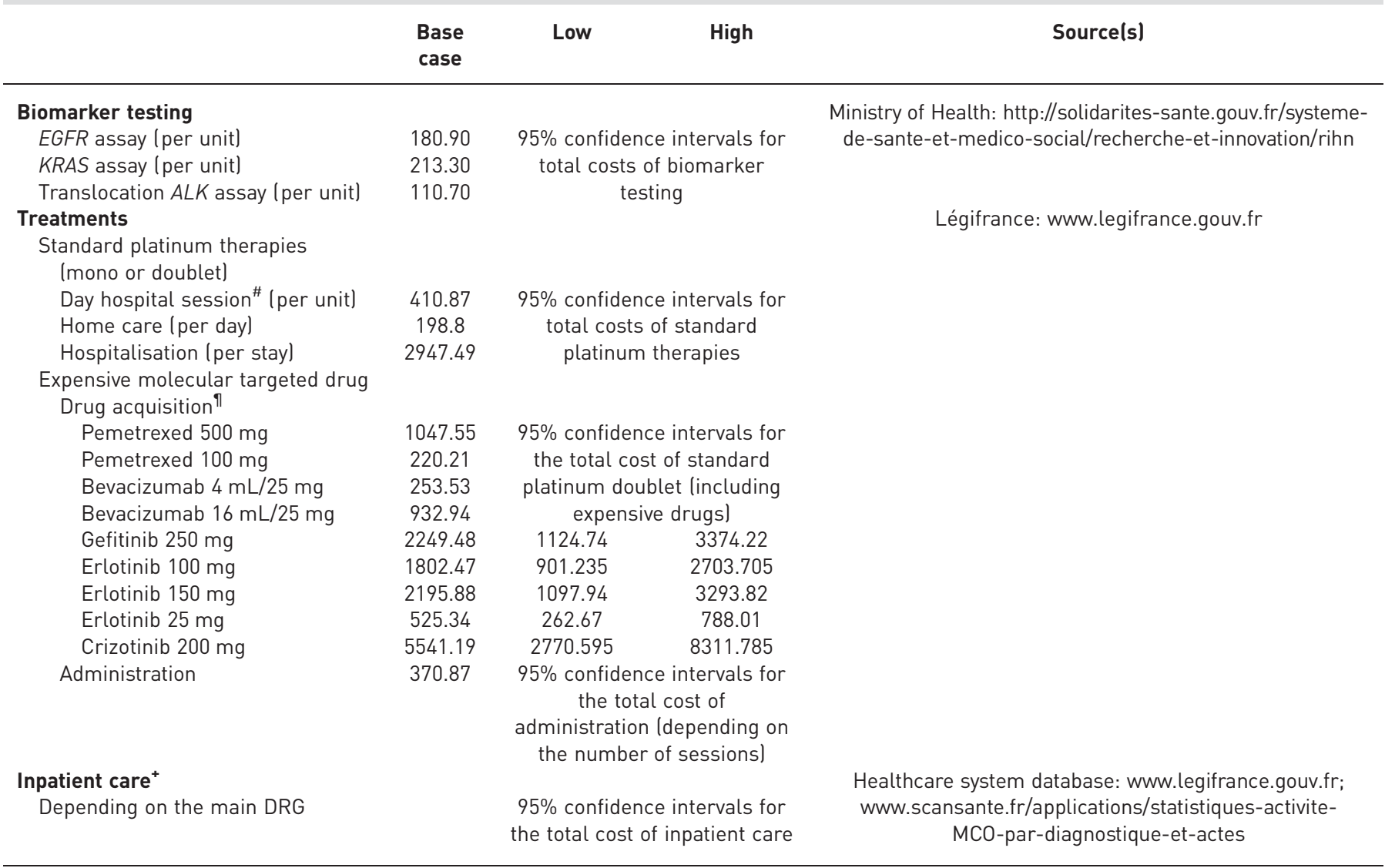

Data are presented as 2015 EUR. DRG: diagnosis-related group. ${ }^{\#}$ : including drugs and administration; ${ }^{\text {I: }}$ public price including tax; ${ }^{+}$: adverse drug event treatment or monitoring according to DRG.

groups exhibited significantly higher BMI and lower performance status, and displayed significantly higher proportions of patients with never-smoker status, brain metastasis and adenocarcinoma histology, and lower use of standard platinum doublet therapies (supplementary table E2), compared with the "no biomarker testing" group.

Each population-based matched analysis included 306 patients (ratio 1:1) (figure 1). Demographic and baseline clinical characteristics were well balanced between the matched groups (table 2).

Cost-effectiveness analysis on first intervention schema: at least one biomarker status known Considering the whole PREDICT.amm cohort $(\mathrm{n}=802)$, mean survival time was 14.9 (95\% CI 13.9-15.7) months in the "at least one biomarker status known" group and 10.6 (95\% CI 9.1-12.1) months in the "no biomarker testing” group (figure 2a).

After matching by propensity score, the "at least one biomarker status known" strategy was still more effective in terms of survival than the control strategy, resulting in a significantly higher survival rate (figure 2b). A total of 0.20 life-years were saved by the "at least one biomarker status known" strategy compared with the "no biomarker testing" strategy (12.94 (95\% CI 11.1-14.5) versus 10.58 (95\% CI 9.04-12.12) months, respectively; p<0.05). Its ICER was EUR13230 per life-year saved (table 3).

Cost-effectiveness analysis on second intervention schema: at least KRAS status known upon first-line therapy decision

The overall survival in the "at least KRAS status known" and "no biomarker testing" groups in the initial population ( $\mathrm{n}=625)$ and the matched study population ( $\mathrm{n}=153$ in each group) is shown in figure $2 \mathrm{c}$ and $\mathrm{d}$. Results of the effectiveness and costs of the matched analysis are given in table 3. In total, 1.93 months 


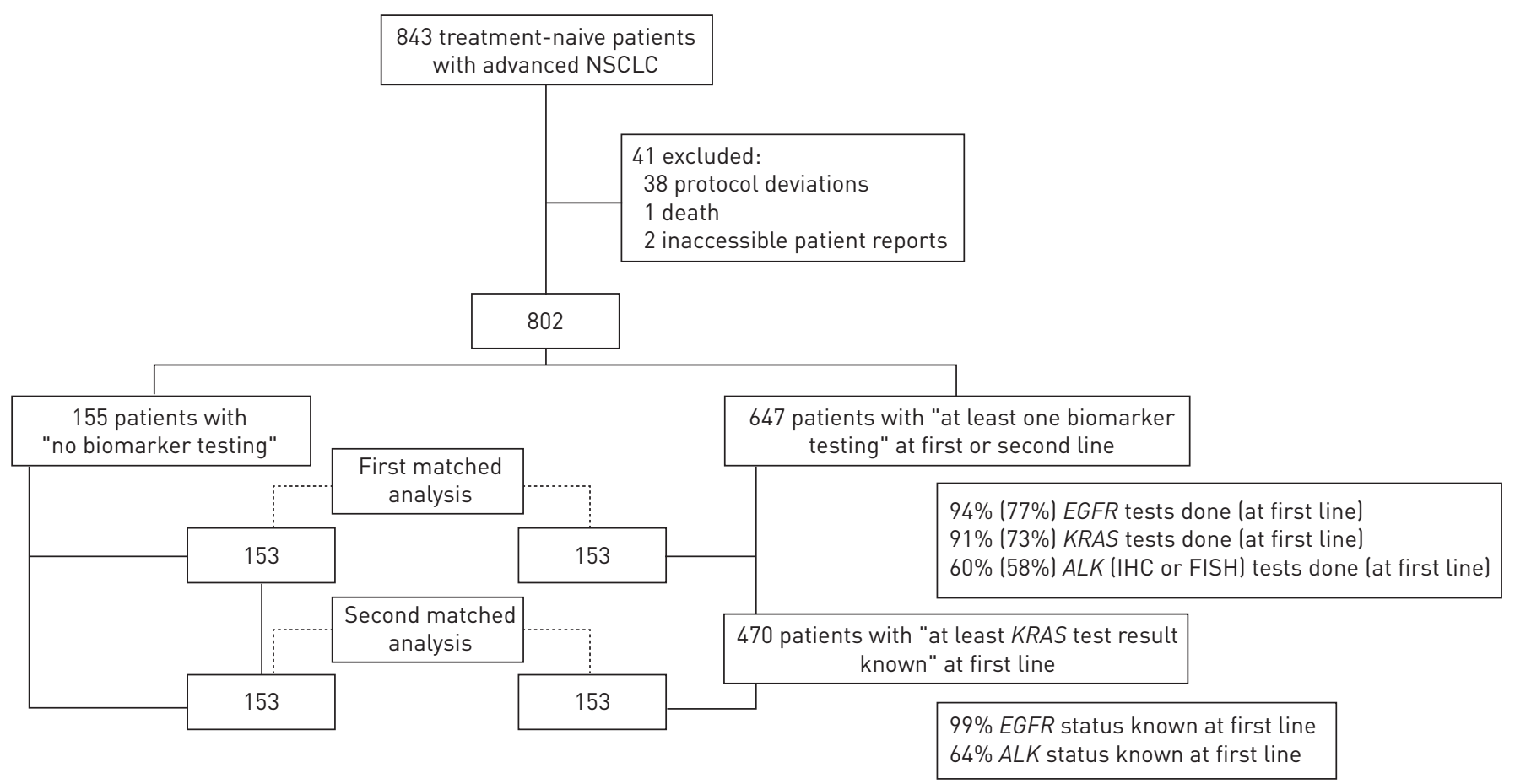

FIGURE 1 Flowchart of patients included in the cost-effectiveness analysis. NSCLC: nonsmall cell lung cancer; IHC: immunohistochemistry; FISH: fluorescence in situ hybridisation.

were saved by the "at least KRAS test result known" strategy. The ICER of the "at least KRAS test result known" strategy compared with the "no biomarker testing” strategy was EUR7444 per life-year saved.

\section{Sensitivity analyses}

The tornado analysis (figure 3) indicated inpatient care and treatment administration costs to be the factors significantly affecting the cost-effectiveness ratio computed when comparing the "at least one

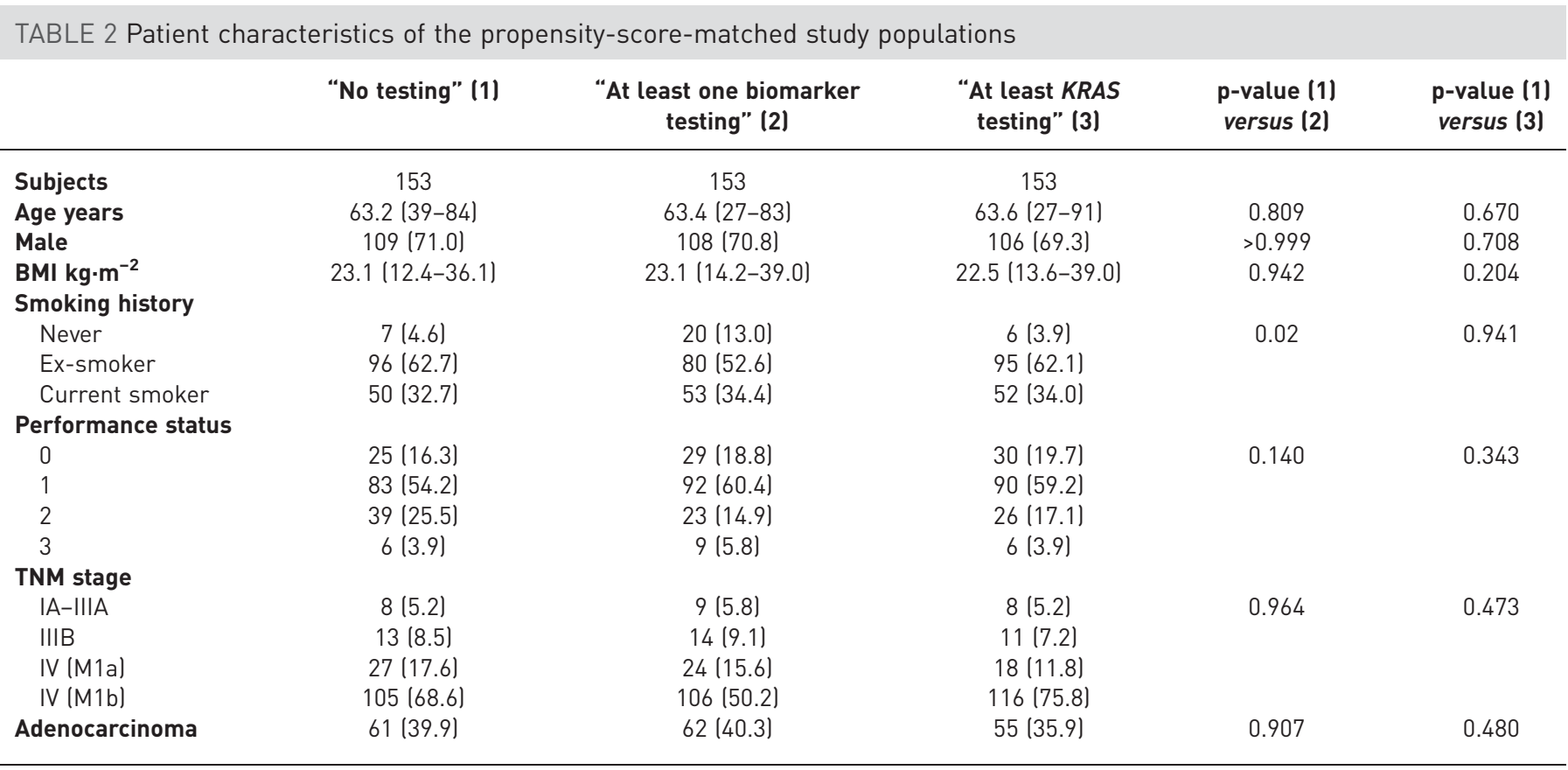

Data are presented as $\mathrm{n}$, mean (minimum-maximum) or $\mathrm{n}(\%)$, unless otherwise stated. BMI: body mass index; TNM: tumour/node/metastasis. 

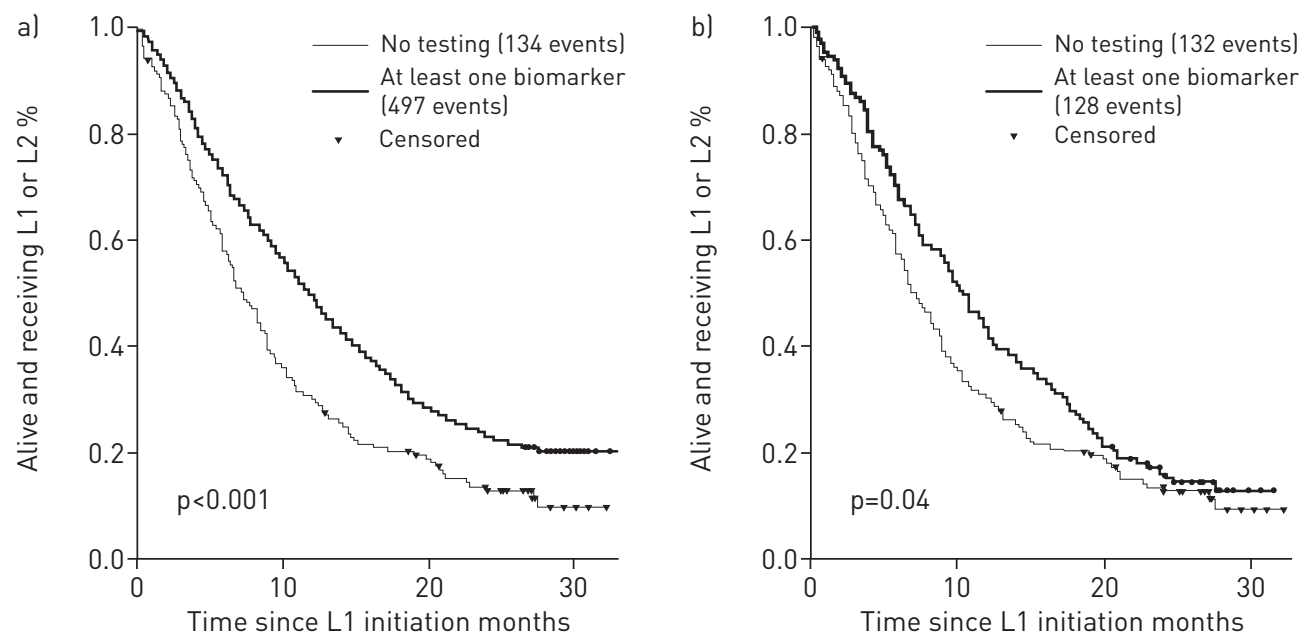

At risk $n$

$\begin{array}{llll}\text { At least one } 647 & 362 & 172 & 15\end{array}$

biomarker

No testing 155

172

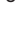

At risk $n$

At least one $153 \quad 38 \quad 31 \quad 2$

biomarker

No testing 153

54

26
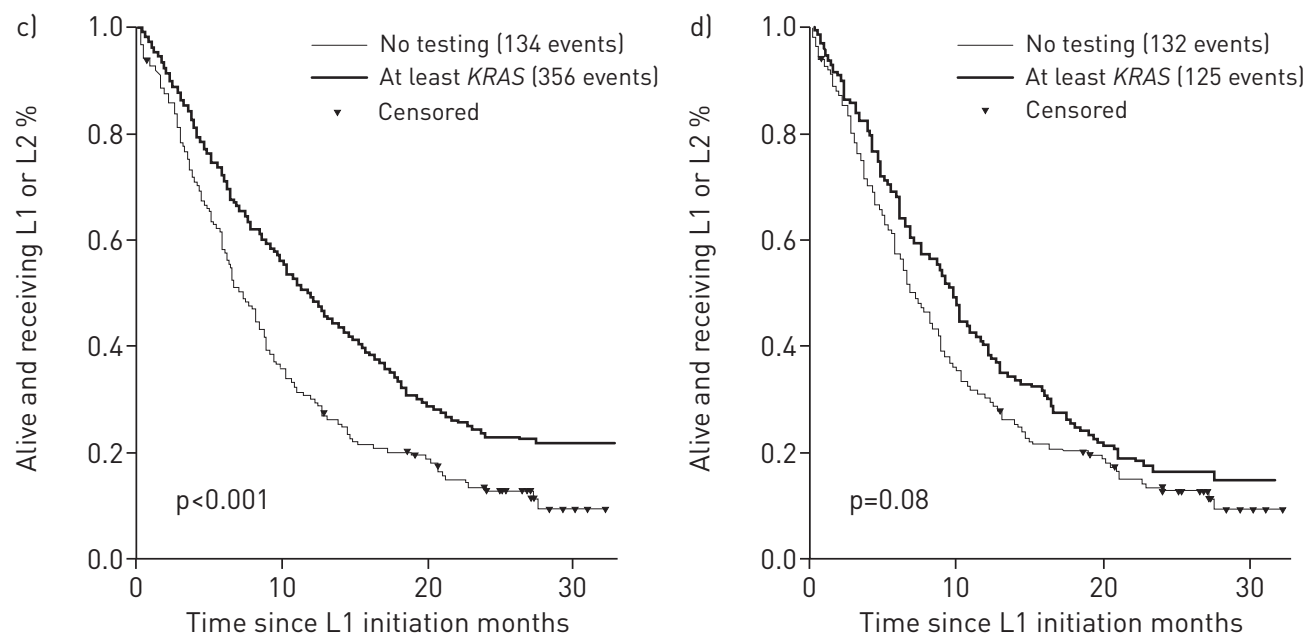

At risk $n$

At least one 470

biomarker

258

No testing 155

258

83

6

53

19

1

At least one 153

biomarker

FIGURE 2 Overall survival for each group (Kaplan-Meier approach). L1: first-line treatment; L2: second-line treatment. a) "At least one biomarker testing" versus "no biomarker testing" in all patients (n=802). b) "At least one biomarker testing" versus "no biomarker testing" in matched populations (n=306). c) "At least KRAS status known" versus "no biomarker testing" in all patients (n=625). d) "At least KRAS status known" versus "no biomarker testing" in matched populations ( $n=153$ ).

biomarker testing" and "no biomarker testing" matched groups. We found the ICER to range from EUR5000 to EUR21500 per life-year saved when inpatient care costs were altered over their 95\% confidence interval. Increasing or decreasing the costs of gefitinib or erlotinib by $50 \%$ had less effect on the ICER. Varying prices for biomarkers testing had only a minor effect on the ICER. To a lesser extent, all other cost parameters also affected the ICER.

Based on the Monte Carlo simulation, $41.0 \%$ of iterations (cost-effect pairs) were located in the southeast quadrant, where the "at least one biomarker status known" intervention would be considered dominant with lower costs and higher effects (figure 4a). However, the scatter plots show a wide variation in the bootstrap estimates. Similarly, the cost-effectiveness acceptability curve indicates that the intervention was cost-effective in $60 \%$ of simulations, at EUR50 000 per life-year saved (figure $4 \mathrm{~b}$ ), indicating that there was 
TABLE 3 Clinical and economic outputs for matched analysis

\begin{tabular}{|c|c|c|c|c|c|}
\hline & “No testing" (1) & $\begin{array}{l}\text { "At least one biomarker } \\
\text { status known" (2) }\end{array}$ & $\begin{array}{c}\text { “At least KRAS status } \\
\text { known" (3) }\end{array}$ & $\begin{array}{l}\mathrm{p} \text {-value (2) } \\
\text { versus (1) }\end{array}$ & $\begin{array}{l}\mathrm{p} \text {-value (3) } \\
\text { versus (1) }\end{array}$ \\
\hline \multicolumn{6}{|l|}{ Effectiveness } \\
\hline PFS months & $6.38(5.26-7.51)$ & $6.58(5.61-7.54)$ & $6.91(5.83-7.99)$ & 0.578 & 0.400 \\
\hline \multicolumn{6}{|l|}{ Costs EUR } \\
\hline Diagnosis & $36(14-58)$ & $513(497-530)$ & $529(519-541)$ & $<0.001$ & $<0.001$ \\
\hline Hospital expensive drugs ${ }^{\pi}$ & $1664(804-2523)$ & $1908(1175-2640)$ & $1774(1187-2361)$ & 0.014 & 0.007 \\
\hline Targeted treatment ${ }^{+}$ & $805(449-1160)$ & 2785 (1732-3838) & $2188(1072-3304)$ & 0.001 & 0.013 \\
\hline Inpatient care $\S^{\S}$ & 10012 (8402-11623) & 8995 (7684-10307) & 8276 (6947-9605) & 0.439 & 0.084 \\
\hline Total costs EUR & 17045 (14861-19228) & 19647 (17 651-21 644) & $18239(16118-20360)$ & 0.006 & 0.133 \\
\hline ICER EUR & & $13320^{f}$ & $7444^{\# \#}$ & & \\
\hline
\end{tabular}

Data presented as mean (95\% Cl) or $\mathrm{n}$, unless otherwise stated. PFS: progression-free survival; ICER: incremental cost-effectiveness ratio. \#: carboplatin or cisplatin in monotherapy or associated with gemcitabine, vinorelbine, docetaxel, etoposide or paclitaxel; П: pemetrexed (Alimta) and bevacizumab (Avastin); ${ }^{+}$: gefitinib (Iressa), erlotinib (Tarceva) and crizotinib (Xalkori); ${ }^{\S}$ : for managing adverse events, disease progression and nonsmall cell lung cancer disease surveillance; ${ }^{f}$ : ICER (2) versus (1); ${ }^{\# \#}$ : ICER (3) versus (1).

some uncertainty associated with the decision regarding cost-effectiveness. In the analysis based on KRAS mutation status, the testing strategy strictly dominated the "no biomarker testing" strategy in $43 \%$ of cases, being cost-effective at EUR50 000 in 58\% of simulations (figure $4 \mathrm{c}$ and d).

\section{Discussion}

Targeted therapies prove effective in patients with specific genetic tumour alterations and it is now well recognised that appropriate patient selection is required. Current European and US guidelines recommend that patients affected by advanced NSCLC receive more individualised therapies based on clinical, histological and molecular results in clinical practice $[2,3]$. Accordingly, the results from the present study conducted in France show routine molecular testing before first- or second-line treatment initiation to be correlated with better survival and limited additional costs. More specifically, our results demonstrate that a strategy assessing the three main genomic alterations (EGFR/KRAS mutations and ALK rearrangement) in advanced NSCLC all-comers, followed by appropriate therapies, either targeted TKI therapies or standard chemotherapy care, is cost-effective compared with a "no biomarker testing" approach along with standard care. With the maximum ICER per life-year saved lying below EUR14000 in the standard case, the testing strategies in question proved cost-effective and are correlated with better ICERs than most cost-effectiveness studies conducted to date in the field of biomarkers testing for lung cancer patients. Such studies, investigating either the testing of one single biomarker $[14,30]$ or multiple biomarkers $[18,31]$, never resulted in an ICER below USD30000 (EUR26400) per life-year saved or quality-adjusted life-year saved. That said, the sensitivity analyses suggest that there is some uncertainty associated with this decision.

In France, public authorities do not refer to a cost-effectiveness threshold to recommend implementation of innovations or to justify reimbursement to manufacturers. The World Health Organization refers to a threshold based on gross domestic product (GDP), an innovative strategy being cost-effective in a developed country with an ICER below three times the GDP per head and very cost-effective with an ICER less that the GDP per head [32]. The French GDP in 2015 was EUR38000 per head, which is well above our base case and worst case ICERs.

Our analysis also revealed that varying the costs of biomarker testing over a plausible population data range did not significantly affect the ICER, whereas the costs of inpatient care and TKI treatment exerted the greatest impact on the cost-effectiveness ratios. Resistance to TKI ultimately developed in almost all patients, although second- and third-generation TKIs have been developed to counteract first-line TKI resistance in EGFR- and ALK-driven NSCLC. Future cost-effectiveness models should thus incorporate a new algorithm for post-treatment monitoring including rebiopsy or circulating tumour DNA into the algorithm for testing and treating patients.

As molecular events are generally exclusive, this study emphasises a cost-effective "testing and treatment" algorithm based on "at least one biomarker testing". Interestingly, the "testing and treatment" algorithm 
a)

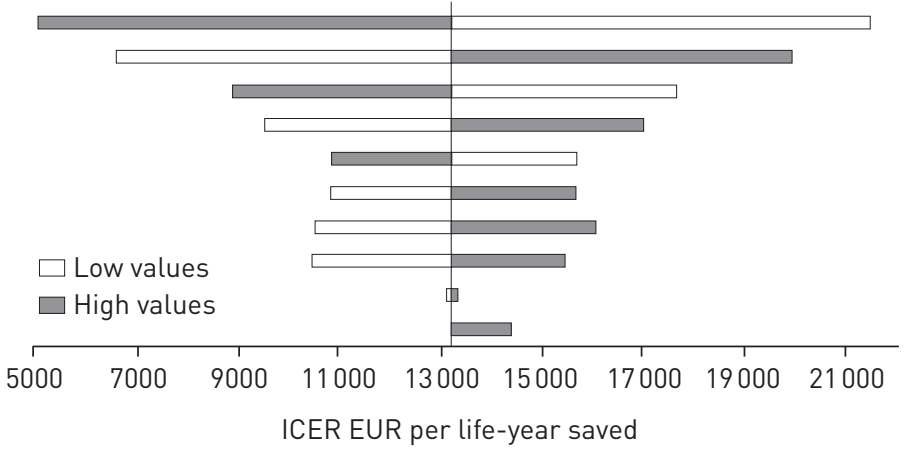

b)

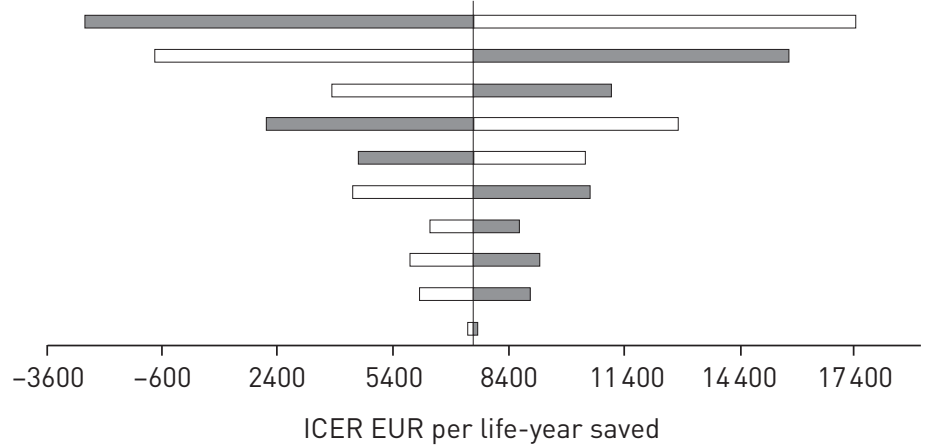

Cost drivers

Inpatient care: control group (lower-upper CI)

Inpatient care: intervention group (lower-upper CI)

Hospital expensive drugs: intervention group (lower-upper Cl)

Hospital expensive drugs: control group (lower-upper Cl)

Standard chemotherapy: control group (lower-upper CI)

Standard chemotherapy: intervention group (lower-upper $\mathrm{Cl}$ )

Erlotinib $(-50 \%$ to $+50 \%)$

Gefitinib (-50\% to $+50 \%)$

Crizotinib $(-50 \%$ to $+50 \%)$

Diagnostic $(95 \% \mathrm{Cl})$

Cost drivers

Inpatient care: control group (lower-upper Cl)

Inpatient care: intervention group (lower-upper $\mathrm{Cl}$ )

Hospital expensive drugs: intervention group (lower-upper Cl)

Hospital expensive drugs: control group (lower-upper Cl)

Standard chemotherapy: control group (lower-upper CI)

Standard chemotherapy: intervention group (lower-upper Cl)

Erlotinib $(-50 \%$ to $+50 \%)$

Gefitinib $(-50 \%$ to $+50 \%)$

Crizotinib $(-50 \%$ to $+50 \%)$

Diagnostic $(95 \% \mathrm{Cl})$

FIGURE 3 Tornado diagrams. Cl: confidence interval; ICER: incremental cost-effectiveness ratio. a) "At least one biomarker testing” versus "no biomarker testing". b) "At least KRAS status known" versus "no biomarker testing".
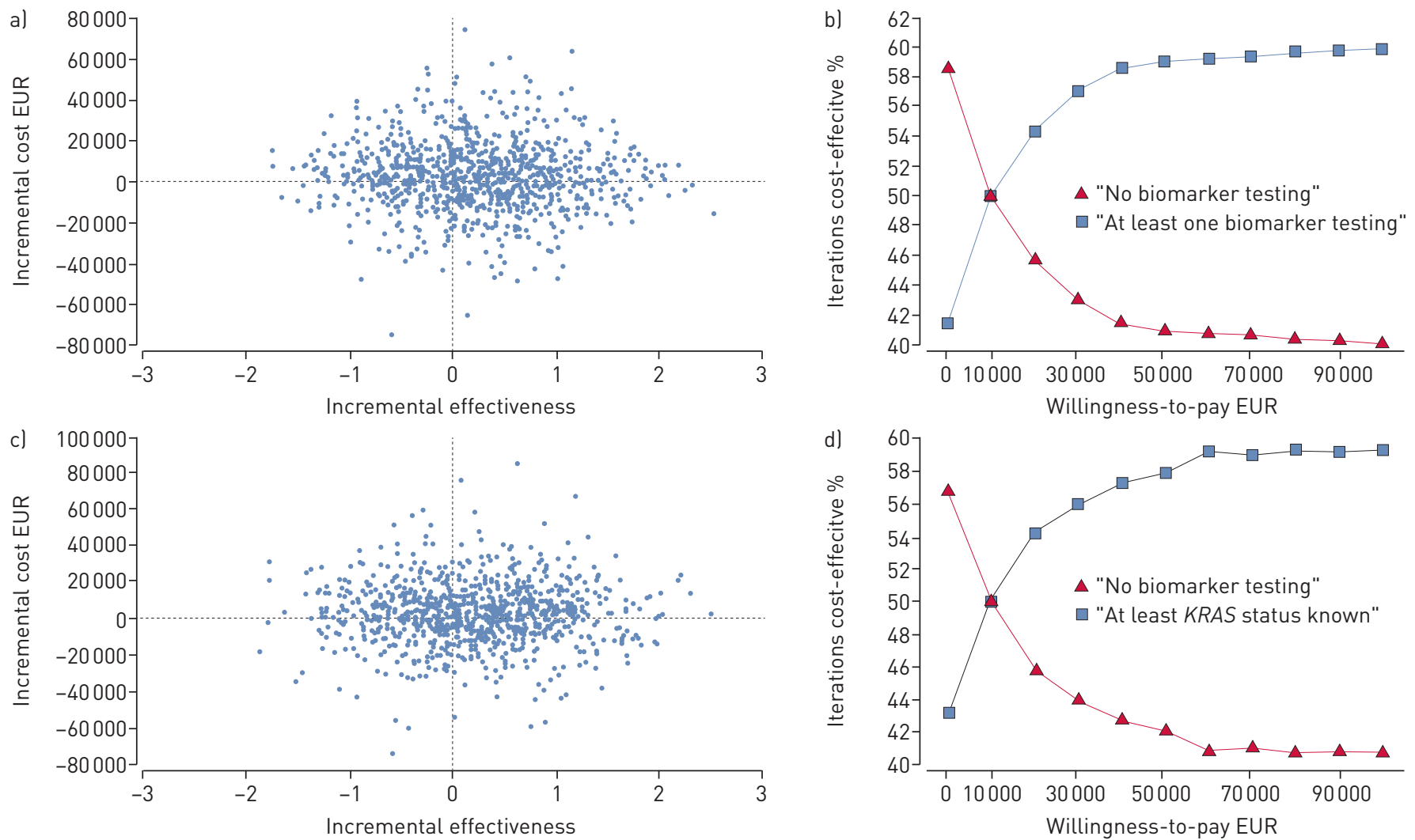

FIGURE 4 Incremental cost-effectiveness scatter plots and acceptability curves in the matched populations. a) Incremental cost-effectiveness scatter plot of sensitivity analysis and b) acceptability curves for "at least one biomarker" versus "no biomarker testing". c) Incremental cost-effectiveness scatter plot of sensitivity analysis and d) acceptability curves for "at least KRAS status known" versus "no biomarker testing". 
based on "at least KRAS testing" proved more cost-effective. While a step-by-step biomarker testing approach was not recommended in France, priority was given to KRAS testing by some centres in the PREDICT.amm cohort. The Biomarkers France study has shown that the presence of KRAS genetic mutations in French patients with NSCLC is of the order of $29 \%$ [1]. To start with KRAS mutation testing appears to be a relevant strategy to optimise the mutually exclusive relationship between KRAS, EGFR and $A L K$ alterations. Indeed, this approach allows much greater cost savings by avoiding unnecessary testing over the patient's lifetime. This result is particularly relevant in countries where the step-by-step testing approach is the intervention of reference and where the cost of biomarkers is mainly borne by the patient.

That said, the risk inherent to this comparatively cost-effective approach would be an increased proportion of patients displaying an unknown oncogenic driver prior to starting first-line treatment due to excessive turnaround time for obtaining the second set of molecular analyses (EGFR and $A L K)[1,33]$. Another risk would be not to identify multiple molecular alterations observed in $\sim 1 \%$ of patients and involving KRAS mutations in $67 \%$ of them. In these mutant KRAS cases, an association with an EGFR mutation or an ALK rearrangement may be observed, which could benefit from a targeted therapy, although less effective in this setting [34].

Thus, up-to-date cost-effectiveness analyses of biomarker testing alternatives yield useful information about the relative interest of future testing approaches. For example, ROS1 (ROS proto-oncogene 1)/ crizotinib and $B R A F^{\mathrm{V} 600} /$ dabrafenib plus trametinib emerge as promising biomarker/therapy couples $[35,36]$, underscoring the potential role of targeted multigene panels in diagnosis and increasing the number of potential molecular events to search for. Finally, the development of NGS strategies, optimised for formalin-fixed, paraffin-embedded and small size samples, could prove to be of assistance in order to validate multigene testing strategies in a routine setting [37, 38]. Evolution of such complex genomic research, enabling multiple concurrent analyses on hundreds of genes, could bear a nonnegligible financial impact, i.e. a potential decrease in costs with NGS versus an increase in costs with circulating DNA monitoring. Recent studies have evaluated the cost-effectiveness of genomic sequencing testing, using mainly decision model approaches [39-41]. Although the patient populations differed among such studies, the overall results showed that compared with "no biomarker testing" or "single biomarker testing", NGS yielded high cost-effectiveness ratios, exceeding USD100000 (EUR88000) per quality-adjusted life-year saved. This thus equates to an ICER over three times the French GDP per head. Our study, as well as most of the cost-effectiveness studies on the diagnosis and treatment of NSCLC patients, showed that treatment costs have a major impact on the global costs of the test-and-treat strategies. By identifying more targetable targets, NGS can result in an increased use of targeted therapies, inducing higher treatment costs. Therefore, it is hard to estimate the future cost impacts of NGS technology, which will depend on the availability of targeted therapies and the reduction in unitary NGS cost and treatment prices. Although NGS appears to be well suited for the molecular characterisation of a growing number of biomarkers in advanced cancer, it is likely that clinicians will use a combination of both step-by-step and NGS strategies in the coming years.

One limitation exhibited by our study is the nonrandomised design. However, we proposed a cohort design in which the "no biomarker testing" group was nested in the prospective cohort for the perspective of the medico-economic analysis. Indeed, all newly diagnosed advanced NSCLC patients in whom an anticancer treatment was initiated for the first time during the study period and followed in the clinical departments participating in the PREDICT.amm project were notified to the study coordinator and therefore included in the study cohort. This point was crucial from the methodological point of view as it avoids the biases associated with a "before" versus "after" approach or with a historical cohort. In addition, our design induced no change in medical practice. Finally, using propensity score matching reduced the potential persistent biases of absence of randomisation. Another limitation is the short follow-up period. Generally, to compensate for the constraints of limited study duration, probabilities of disease progression and costs were extrapolated over the course of a patient's lifetime based on data from literature and derived hypotheses. That said, such a model approach could entail potential sources of bias (stemming from hypotheses) and final results could differ from the observed clinical practice (not based on prospective, observational population data). In fact, several studies have demonstrated that a 2-year follow-up period proves sufficiently long to capture the major health and economic consequences presented by metastatic cancer $[18,42]$. It should be noted that $80 \%$ of our study patients died before the end of the 2-year follow-up period.

Acknowledgements: The authors are grateful to all the investigators for their participation in the study. We thank Cécile Fortanier (APHM, Marseille, France) who contributed to the study design and the grant proposal, and Quân Tran, Antoine Deroy and Sandy Dos Santos (all IFCT, Paris, France) for their participation in data collection, monitoring and computing. 
Conflict of interest: D. Moro-Sibilot has received personal fees from Eli Lilly, Roche, Pfizer, Ariad, Boehringer, AstraZeneca, Novartis, MSD and BMS, outside the submitted work. S. Friard has received nonfinancial support from Roche, AstraZeneca, BMS, Boehringer, Lilly, Novartis and Pfizer, outside the submitted work. M. Wislez has received grants from BMS Fondation, nonfinancial support from Roche, personal fees and nonfinancial support from Lilly and Boehringer Ingelheim, and personal fees from AstraZeneca, MSD and BMS, outside the submitted work. V. Westeel has received personal fees (speaker's bureau, advisory role, meeting expenses) from Lilly, personal fees (speaker's bureau, advisory role, meeting expenses, research funding) from Roche, personal fees (speaker's bureau, advisory role) from AstraZeneca and personal fees (speaker's bureau, meeting expenses, research funding) from Boehringer Ingelheim, outside the submitted work. H. Léna has received grants and nonfinancial support from Roche, personal fees from AstraZeneca, Merck, Pierre Fabre Oncology, Bristol-Myers Squibb and Boehringer Ingelheim, and personal fees and nonfinancial support from Pfizer and Lilly, outside the submitted work. G. Zalcman has received grants, personal fees and acted as an investigator in clinical trials for Roche and Pfizer, and has acted as an investigator in clinical trials for AstraZeneca, Boehringer and GSK, during the conduct of the study; and has received grants and acted as an investigator in clinical trials for BMS and Roche, outside the submitted work. M. Antoine has received personal fees (for board participation) from Roche and MSD, outside the submitted work. F. Barlesi has received personal fees from AstraZeneca, Bristol-Myers Squibb, Boehringer Ingelheim, Clovis Oncology, Eli Lilly Oncology, F. Hoffmann-La Roche Ltd, Novartis, Merck, MSD, Pierre Fabre and Pfizer, outside the submitted work. J. Cadranel has received grants (for research in ALK immune response) and personal fees (for participation on the board of clinical trials) from Pfizer, grants (for research in NGS) and personal fees (for participation on the board of clinical trials) from Novartis and AstraZeneca, and personal fees (for participation on the board of clinical trials) from Roche, Boehringer Ingelheim, BMS and Lilly, outside the submitted work.

Support statement: This work was supported by an institutional grant from the French Programme Hospitalier Recherche Clinique National (PRME 2009) - French Ministry of Health, French National Cancer Institute (INCa), French League Against Cancer, Roche, GSK, Boehringer Ingelheim and Lilly-AstraZeneca. These funding sources had no role in the design of this study and will not have any role during its execution, analyses, interpretation of the data or decision to submit results. The sponsor was represented by Assistance Publique Hôpitaux de Paris, France; and its role was to control the appropriateness of ethical and legal considerations. Funding information for this article has been deposited with the Crossref Funder Registry.

\section{References}

1 Barlesi F, Mazieres J, Merlio JP, et al. Routine molecular profiling of patients with advanced non-small-cell lung cancer: results of a 1-year nationwide programme of the French Cooperative Thoracic Intergroup (IFCT). Lancet 2016; 387: 1415-1426.

2 National Comprehensible Cancer Network. Clinical Practice Guidelines in Oncology. Fort Washington, NCCN, 2015.

3 European Society for Medical Oncology. Clinical Practice Guidelines: Lung and Chest Tumours Viganello-Lugano, ESMO, 2016.

4 Rosell R, Carcereny E, Gervais R, et al. Erlotinib versus standard chemotherapy as first-line treatment for European patients with advanced EGFR mutation-positive non-small-cell lung cancer (EURTAC): a multicentre, open-label, randomised phase 3 trial. Lancet Oncol 2012; 13: 239-246.

5 Yang JC, Wu YL, Schuler M, et al. Afatinib versus cisplatin-based chemotherapy for EGFR mutation-positive lung adenocarcinoma (LUX-Lung 3 and LUX-Lung 6): analysis of overall survival data from two randomised, phase 3 trials. Lancet Oncol 2015; 16: 141-151.

6 Mok TS, Wu YL, Thongprasert S, et al. Gefitinib or carboplatin-paclitaxel in pulmonary adenocarcinoma. $N$ Engl J Med 2009; 361: 947-957.

7 Linardou H, Dahabreh IJ, Kanaloupiti D, et al. Assessment of somatic $k$-RAS mutations as a mechanism associated with resistance to EGFR-targeted agents: a systematic review and meta-analysis of studies in advanced non-small-cell lung cancer and metastatic colorectal cancer. Lancet Oncol 2008; 9: 962-972.

8 Pao W, Wang TY, Riely GJ, et al. KRAS mutations and primary resistance of lung adenocarcinomas to gefitinib or erlotinib. PLoS Med 2005; 2: e17.

9 Roberts PJ, Stinchcombe TE, Der CJ, et al. Personalized medicine in non-small-cell lung cancer: is KRAS a useful marker in selecting patients for epidermal growth factor receptor-targeted therapy? J Clin Oncol 2010; 28: 4769-4777.

10 Soria JC, Tan DS, Chiari R, et al. First-line ceritinib versus platinum-based chemotherapy in advanced $A L K$-rearranged non-small-cell lung cancer (ASCEND-4): a randomised, open-label, phase 3 study. Lancet 2017; 389: 917-929.

11 Shaw AT, Kim DW, Nakagawa K, et al. Crizotinib versus chemotherapy in advanced ALK-positive lung cancer. N Engl J Med 2013; 368: 2385-2394.

12 Solomon BJ, Mok T, Kim DW, et al. First-line crizotinib versus chemotherapy in ALK-positive lung cancer. $N$ Engl J Med 2014; 371: 2167-2177.

13 Kris MG, Johnson BE, Berry LD, et al. Using multiplexed assays of oncogenic drivers in lung cancers to select targeted drugs. JAMA 2014; 311: 1998-2006.

14 Arrieta O, Anaya P, Morales-Oyarvide V, et al. Cost-effectiveness analysis of EGFR mutation testing in patients with non-small cell lung cancer (NSCLC) with gefitinib or carboplatin-paclitaxel. Eur J Health Econ 2016; 17: $855-863$

15 Borget I, Cadranel J, Pignon JP, et al. Cost-effectiveness of three strategies for second-line erlotinib initiation in nonsmall-cell lung cancer: the ERMETIC study part 3. Eur Respir J 2012; 39: 172-179.

16 Carlson JJ, Reyes C, Oestreicher N, et al. Comparative clinical and economic outcomes of treatments for refractory non-small cell lung cancer (NSCLC). Lung Cancer 2008; 61: 405-415.

17 Lewis G, Peake M, Aultman R, et al. Cost-effectiveness of erlotinib versus docetaxel for second-line treatment of advanced non-small-cell lung cancer in the United Kingdom. J Int Med Res 2010; 38: 9-21. 
18 Romanus D, Cardarella S, Cutler D, et al. Cost-effectiveness of multiplexed predictive biomarker screening in non-small-cell lung cancer. J Thorac Oncol 2015; 10: 586-594.

19 Loubiere S, Moatti JP. Economic evaluation of point-of-care diagnostic technologies for infectious diseases. Clin Microbiol Infect 2010; 16: 1070-1076.

20 McLeer-Florin A, Moro-Sibilot D, Melis A, et al. Dual IHC and FISH testing for ALK gene rearrangement in lung adenocarcinomas in a routine practice: a French study. J Thorac Oncol 2012; 7: 348-354.

21 Beau-Faller $\mathrm{M}$, Blons $\mathrm{H}$, Domerg $\mathrm{C}$, et al. A multicenter blinded study evaluating EGFR and KRAS mutation testing methods in the clinical non-small cell lung cancer setting - IFCT/ERMETIC2 Project Part 1: comparison of testing methods in 20 French molecular genetic National Cancer Institute platforms. J Mol Diagn 2014; 16: 45-55.

22 Beau-Faller M, Degeorges A, Rolland E, et al. Cross-validation study for epidermal growth factor receptor and KRAS mutation detection in 74 blinded non-small cell lung carcinoma samples: a total of 5550 exons sequenced by 15 molecular French laboratories (evaluation of the EGFR mutation status for the administration of EGFR-TKIs in non-small cell lung carcinoma [ERMETIC] project - part 1). J Thorac Oncol 2011; 6: 1006-1015.

23 Blons $\mathrm{H}$, Rouleau E, Charrier N, et al. Performance and cost efficiency of KRAS mutation testing for metastatic colorectal cancer in routine diagnosis: the MOKAECM study, a nationwide experience. PLoS One 2013; 8: e68945.

24 Riely GJ, Marks J, Pao W. KRAS mutations in non-small cell lung cancer. Proc Am Thorac Soc 2009; 6: 201-205.

25 Husereau D, Drummond M, Petrou S, et al. Consolidated Health Economic Evaluation Reporting Standards (CHEERS) statement. Pharmacoeconomics 2013; 31: 361-367.

26 Austin PC. The use of propensity score methods with survival or time-to-event outcomes: reporting measures of effect similar to those used in randomized experiments. Stat Med 2014; 33: 1242-1258.

27 Karim ME, Gustafson P, Petkau J, et al. Comparison of statistical approaches for dealing with immortal time bias in drug effectiveness studies. Am J Epidemiol 2016; 184: 325-335.

28 Barton GR, Briggs AH, Fenwick EA. Optimal cost-effectiveness decisions: the role of the cost-effectiveness acceptability curve (CEAC), the cost-effectiveness acceptability frontier (CEAF), and the expected value of perfection information (EVPI). Value Health 2008; 11: 886-897.

29 Fenwick E, Claxton K, Sculpher M. Representing uncertainty: the role of cost-effectiveness acceptability curves. Health Econ 2001; 10: 779-787.

30 Narita Y, Matsushima Y, Shiroiwa T, et al. Cost-effectiveness analysis of EGFR mutation testing and gefitinib as first-line therapy for non-small cell lung cancer. Lung Cancer 2015; 90: 71-77.

31 Doble B, John $\mathrm{T}$, Thomas $\mathrm{D}$, et al. Cost-effectiveness of precision medicine in the fourth-line treatment of metastatic lung adenocarcinoma: an early decision analytic model of multiplex targeted sequencing. Lung Cancer 2017; 107: 22-35.

32 World Health Organization. Making choices in health: WHO guide to cost-effectiveness analysis. 2003. http://apps.who.int/iris/bitstream/10665/42699/1/9241546018.pdf Date last accessed: February 22, 2018

33 Lim C, Tsao MS, Le LW, et al. Biomarker testing and time to treatment decision in patients with advanced nonsmall-cell lung cancer. Ann Oncol 2015; 26: 1415-1421.

34 Guibert N, Barlesi F, Descourt R, et al. Characteristics and outcomes of patients with lung cancer harboring multiple molecular alterations: results from the IFCT Study Biomarkers France. J Thorac Oncol 2017; 12: 963-973.

35 Mazieres J, Zalcman G, Crino L, et al. Crizotinib therapy for advanced lung adenocarcinoma and a ROS1 rearrangement: results from the EUROS1 cohort. J Clin Oncol 2015; 33: 992-999.

36 Planchard D, Besse B, Groen HJ, et al. Dabrafenib plus trametinib in patients with previously treated $B R A F^{\mathrm{V} 600 \mathrm{E}}$-mutant metastatic non-small cell lung cancer: an open-label, multicentre phase 2 trial. Lancet Oncol 2016; 17: 984-993.

37 Fallet V, Saffroy R, Girard N, et al. High-throughput somatic mutation profiling in pulmonary sarcomatoid carcinomas using the LungCarta Panel: exploring therapeutic targets. Ann Oncol 2015; 26: 1748-1753.

38 Kaisaki PJ, Cutts A, Popitsch N, et al. Targeted next-generation sequencing of plasma DNA from cancer patients: factors influencing consistency with tumour DNA and prospective investigation of its utility for diagnosis. PLoS One 2016; 11: e0162809.

39 Christensen KD, Dukhovny D, Siebert U, et al. Assessing the costs and cost-effectiveness of genomic sequencing. J Pers Med 2015; 5: 470-486.

40 Sabatini LM, Mathews C, Ptak D, et al. Genomic sequencing procedure microcosting analysis and health economic cost-impact analysis: a report of the association for molecular pathology. J Mol Diagn 2016; 18: $319-328$

41 van Amerongen RA, Retel VP, Coupe VM, et al. Next-generation sequencing in NSCLC and melanoma patients: a cost and budget impact analysis. Ecancermedicalscience 2016; 10: 684 .

42 Djalalov S, Beca J, Hoch JS, et al. Cost effectiveness of EML4-ALK fusion testing and first-line crizotinib treatment for patients with advanced ALK-positive non-small-cell lung cancer. J Clin Oncol 2014; 32: 1012-1019. 